\title{
Cardiopulmonary resuscitation with assisted extracorporeal life support during cardiac arrest caused by drug-eluting stent thrombosis
}

-a case report-

\author{
Ju-Hyun Lee ${ }^{1}$, Sang-Heon Park ${ }^{2}$, and In Ae Song ${ }^{2}$ \\ Department of Anesthesiology and Pain Medicine, ${ }^{1}$ Seoul National University Hospital, Seoul, ${ }^{2}$ Seoul National University Bundang \\ Hospital, Seongnam, Korea
}

Discontinuation of dual antiplatelet therapy within 12 months after drug-eluting stent (DES) implantation increases the possibility of stent thrombosis. We now report the case of a 66-year-old man who suffered a cardiac arrest due to stent thrombosis after an elective laparoscopic anterior resection. Ten month ago, he underwent DES implantation and then had been taking dual antiplatelet therapy. Nine days prior to the surgery, he discontinued dual antiplatelet therapy. Forty minutes after intensive care unit admission, cardiac arrest occurred. However, his cardiac rhythm did not restore in spite of resuscitation, so immediately veno-arterial extracorporeal membrane oxygenation (ECMO) was implanted. Four days after the surgery, he was weaned from ECMO support, recovered completely, with no cardiopulmonary or neurological sequelae. (Korean J Anesthesiol 2014; 66: 383-387)

Key Words: Drug-eluting stents, Extracorporeal membrane oxygenation, Thrombosis.

Drug-eluting stent (DES) has been mostly used as percutaneous coronary intervention (PCI) and the number of noncardiac surgery cases after DES implantation increases. However, noncardiac surgery after coronary stent implantation increases the risks of stent thrombosis, myocardial infarction (MI), and so on [1-3]. Therefore, dual antiplatelet therapy is recommended for 12 months $[1,3,4]$; however, since it would cause additional bleeding during surgery, dual antiplatelet therapy tends to be discontinued 5 to 10 days prior to surgery $[2,4]$.
We experienced an adult patient who discontinued dual antiplatelet therapy within 12 months after DES implantation and suffered from sudden cardiac arrest. Veno-arterial extracorporeal membrane oxygenation (ECMO) was immediately used 7 minutes after cardiopulmonary resuscitation (CPR). He was diagnosed with stent thrombosis and successfully weaned from the support completely without cardiopulmonary or neurological sequelae.

Received: February 14, 2013. Revised: April 8, 2013. Accepted: April 22, 2013.

Corresponding author: Sang-Heon Park, M.D., Department of Anesthesiology and Pain Medicine, Seoul National University Bundang Hospital, 300, Gumi-dong, Bundang-gu, Seongnam 463-802, Korea. Tel: 82-31-787-7499, Fax: 82-31-787-4063, E-mail: psh1399@snubh.org

(c) This is an open-access article distributed under the terms of the Creative Commons Attribution Non-Commercial License (http:// creativecommons.org/licenses/by-nc/3.0/), which permits unrestricted non-commercial use, distribution, and reproduction in any medium, provided the original work is properly cited. 


\section{Case Report}

A 66-year-old male patient (height $170.7 \mathrm{~cm}$, body weight $64.9 \mathrm{~kg}$ ) was scheduled for a laparoscopic anterior resection due to sigmoid colon cancer. He had a history of hypertension, diabetes mellitus, angina, and congestive heart failure. Ten months ago, he was diagnosed with MI. Hence, DES were implanted for left anterior descending (LAD) and left circumflex (LCX) coronary artery stenoses. He had been taking aspirin, clopidogrel, statin, angiotensin-converting enzyme inhibitor, furosemide, spironolactone and thiazide. Although a cardiologist recommended that medications should be stopped within 7 days, administration of aspirin and clopidogrel was voluntarily stopped 9 days before the surgery. However, the operation could be postponed no longer. Thus, his family sufficiently listened to the explanations about the state of patient, very high risk for perioperative MI, heart failure or sudden death and agreed to the surgery.

Preoperative laboratory results were as follows; electrocardiogram (ECG) information showed atrial fibrillations (Afib) and the echocardiography showed ischemic cardiomyopathy with severe left ventricular dysfunction, ejection fraction $24 \%$, and regional wall motion abnormality (akinesia at whole apex, mid anterior, anteroseptum, basal inferior, basal to mid posterior, and mid lateral). Laboratory studies were unremarkable, but pulmonary edema was diagnosed on chest X-ray and the pulmonary function testing showed a mild restrictive pattern $(\mathrm{FEV} 1 / \mathrm{FVC}=64 \%)$.

Emergency drugs and defibrillator were ready at the operating room for sudden blood pressure (BP) drop or cardiac arrest. Premedication was not used. Monitoring were included 3-lead ECG with continuous ST segment analysis, pulse oximetry $\left(\mathrm{SpO}_{2}\right)$, non-invasive BP monitor, and end-tidal carbon dioxide $\left(\mathrm{EtCO}_{2}\right)$ measurements. Before anesthesia, vital signs included a BP of 113/63 mmHg, a heart rate (HR) of 88 beats/min, and $\mathrm{SpO}_{2}$ of $98 \%$. After Allen's test, an arterial catheter was inserted in the right radial artery for continuous BP monitoring. After preoxygenation with $100 \% \mathrm{O}_{2}$, etomidate $14 \mathrm{mg}$ and rocuronium 50 mg were administered intravenously. Remifentanil was continuously infused using a TCI device (Orchestra ${ }^{\circledR}$ Base Primea, Fresenius-Vial, Brezins, France) with $2 \mathrm{ng} / \mathrm{ml}$ at the effect-site concentration. Anesthesia was maintained using $0.7 \mathrm{~L} / \mathrm{min} \mathrm{O}_{2}$, $1.3 \mathrm{~L} / \mathrm{min}$ air, $1.5-2.5 \mathrm{vol} \%$ sevoflurane, and remifentanil 1.0-2.0 $\mathrm{ng} / \mathrm{ml}$. Dopamine at $0-7 \mu \mathrm{g} / \mathrm{kg} / \mathrm{min}$ was continuously infused to maintain the mean BP above $60 \mathrm{mmHg}$. Ventilation was mechanically performed with a tidal volume of $480 \mathrm{ml}$ and a rate of 12 breaths/min, and $\mathrm{EtCO}_{2}$ was kept between 31 and $45 \mathrm{mmHg}$.

The operative technique involved intraperitoneal insufflation of $\mathrm{CO}_{2}$. The patient was placed in the Trendelenburg position and pneumoperitoneum was maintained with a variable-flow insufflator at a pressure of $13 \mathrm{mmHg}$ throughout the surgery. Twelve minutes after the Trendelenburg position, the ST segment level decreased from initially -0.3 to -1.0 , so nitroglycerin (NTG) $0.5 \mu \mathrm{g} / \mathrm{kg} / \mathrm{min}$ was continuously infused. When $\mathrm{CO}_{2}$ insufflation was stopped, the ST segment level was restored to the initial level. Thereafter, the NTG infusion was stopped due to low BP. The operation was completed without any other significant complications and the patient was transferred to an intensive care unit (ICU). The total operation time was 1 hour and 55 minutes and the patient was under anesthesia for 3 hours. Crystalloid $250 \mathrm{ml}$ and colloid $500 \mathrm{ml}$ were totally infused. The estimated blood loss was $30 \mathrm{ml}$, and the urine output was $250 \mathrm{ml}$.

On arrival at the ICU, the patient's vital signs were stable. Forty minutes after transfer to the ICU, sudden ventricular premature complexes occurred at a rate above 50 per minute, so 2 times of lidocaine $70 \mathrm{mg}$ were injected. Ten minutes after the injection, a sudden onset of ventricular tachycardia was noted on the ECG. Synchronized electrical cardioversion at $150 \mathrm{~J}$ was performed. Thereafter, chest compressions were given and then epinephrine $1 \mathrm{mg}$ and atropine $0.5 \mathrm{mg}$ were intravenously injected. Four minutes after resuscitation, this was followed by ventricular fibrillation, epinephrine $1 \mathrm{mg}$ and defibrillation with 200 J. When the compression stopped, the cardiac rhythm was not restored. Epinephrine $0.1 \mu \mathrm{g} / \mathrm{kg} / \mathrm{min}$ was continuously infused and we decided to perform veno-arterial ECMO insertion 7 minutes after CPR. During ECMO insertion, chest compression was continuously performed, including defibrillation $200 \mathrm{~J}$, and epinephrine $1 \mathrm{mg}$ and sodium bicarbonate $40 \mathrm{mEq}$ injection two times. Twenty three minutes after cardiac arrest, ECMO support was applied and a 12-lead portable ECG (Fig. 1) was checked. Nevertheless, the ventricular fibrillation continued and defibrillation at $200 \mathrm{~J}$ was performed 4 times. Due to the lack of a response to $100 \mathrm{mg}$ injected lidocaine, an amiodarone infusion was started. Epinephrine dosage was increased to $0.15 \mu \mathrm{g} / \mathrm{kg} /$

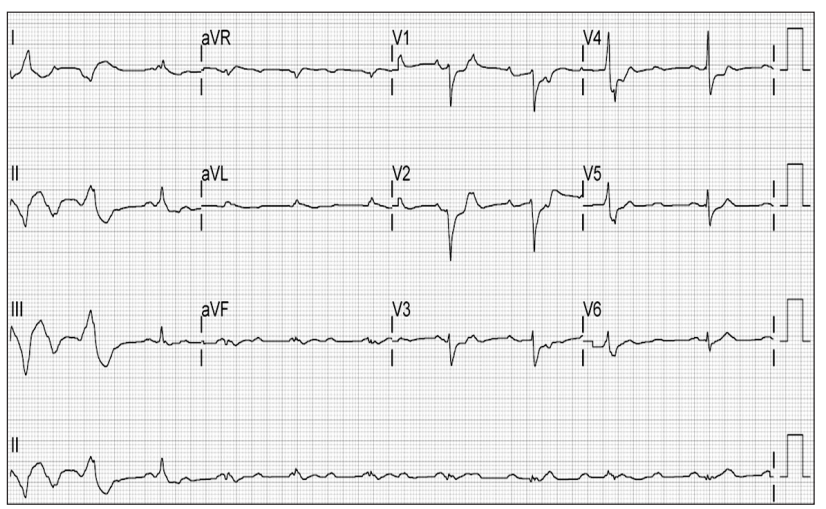

Fig. 1. The 12-lead portable electrocardiogram shows atrial fibrillation, anterior infarct, nonspecific intraventricular conduction delay right after extracorporeal membrane oxygenation insertion. 
min and dopamine $10 \mu \mathrm{g} / \mathrm{kg} / \mathrm{min}$ was continuously infused.

Transthoracic echocardiography showed lower cardiac output $(\mathrm{EF}<15 \%)$ and wider anterior wall motion abnormality than preoperative finding. According to abnormal transthoracic echocardiographic findings and the patient's medical history, anterior infarction caused by stent thrombosis was strongly suspicious. Thus, coronary angiography was performed. The patient was transferred to the cardiac catheterization room with ECMO support and dopamine $10 \mu \mathrm{g} / \mathrm{kg} / \mathrm{min}$, amiodarone 0.5 $\mathrm{mg} / \mathrm{min}$, heparin $10 \mathrm{u} / \mathrm{kg} / \mathrm{hr}$, and epinephrine $0.05 \mu \mathrm{g} / \mathrm{kg} / \mathrm{min}$ administration. The angiographic findings showed that stent thromboses were detected in both LAD and LCX stents (Fig. 2). In addition, total occlusion of the proximal right coronary artery was detected. Therefore, balloon inflation was performed in the LAD and the LCX. In addition, intraaortic balloon pump (IABP) was inserted in the left femoral artery.

In the ICU, vital signs were stable at ECMO flow rates between 3 and $4 \mathrm{~L} / \mathrm{min}$ and the ECG was normal sinus rhythm. Therefore, the dosages of dopamine and epinephrine were reduced to $5 \mu \mathrm{g} / \mathrm{kg} / \mathrm{min}$ and $0.01 \mu \mathrm{g} / \mathrm{kg} / \mathrm{min}$ each. Heparin $6 \mathrm{u} / \mathrm{kg} / \mathrm{hr}$, aspirin $100 \mathrm{mg}$ and clopidogrel $75 \mathrm{mg}$ were administered as anticoagulants.

On the 3rd day after the surgery, the vital signs were stable. Therefore, he was weaned from ECMO support. On the 5th day after the surgery, the patient was extubated, and IABP was removed on the 6th day. On the 7th day, the portable transthoracic echocardiography showed that estimated left ventricular ejection fraction was 30 to $35 \%$ and the ECG showed normal sinus rhythm. Because the patient had stable vital signs without inotropics and vasopressors, the patient was transferred to the

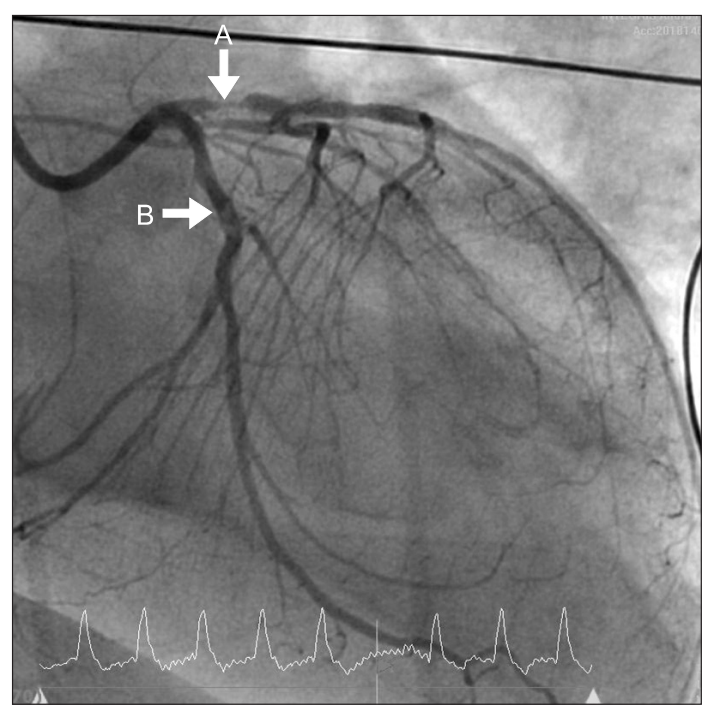

Fig. 2. Coronary angiography: The angiographic finding shows stent thromboses in both left anterior descending (A) and left circumflex stents (B). general ward, and discharged from the hospital on the 36th day after the surgery.

\section{Discussion}

Since the introduction of DES implantation, the number of noncardiac surgical procedures after performed DES implantation is progressively increasing. However, patients who undergo noncardiac surgery after DES implantation have high perioperative risks of mortality induced by myocardial ischemia, MI and acute coronary syndrome (ACS) [1-3]. Therefore, after DES implantation, it is recommended to continue dual antiplatelet therapy for a minimum of 12 months, and noncardiac surgery shall be postponed during this period. If surgery is necessary within 12 months of dual antiplatelet therapy, the therapy shall not be discontinued $[1,3,4]$. It is critical that patients undergoing DES should continue dual antiplatelet therapy for at least 1 year, because stent thrombosis is a catastrophic complication in percutaneous revascularization procedures that results in MI with $45 \%$ mortality rate $[3,4]$.

The key predictors of stent thrombosis were premature antiplatelet therapy discontinuation, renal failure, bifurcation lesion, diabetes mellitus, and low ejection fraction. For stent length, subacute thrombosis was also a predictor, and the most important independent predictor is premature discontinuation of dual antiplatelet therapy, leading to $1.3 \%$ stent thrombosis $[3,4]$. A prospective study reported that stent thrombosis incidence 9 months after DES implantation was 90 times higher in patients who discontinued dual antiplatelet therapy prematurely than who continued [4]. In addition, in the occurrence of stent endothelialization, continuous administration of dual antiplatelet therapy, such as aspirin and clopidogrel, reduces the risk of stent thrombosis below 1\% [5].

On the other hand, antiplatelet therapy changes the principal functions of platelets and increases the risk of surgical bleeding. In the case of the laparoscopic surgery, it has narrow confines of the surgical field [6], which can be disturbed by small bleeding. Therefore, due to the possibility of excessive bleeding, dual antiplatelet therapy has been normally discontinued for a few days prior to surgery [4]. Nevertheless, no study has clearly indicated that discontinuation of perioperative antiplatelet therapy directly leads to excessive surgical bleeding [5,7], and according to Chassot et al. [8], if antiplatelet drugs are continued, aspirin alone increases bleeding by $2.5-20 \%$ and combination of aspirin and clopidogrel increases bleeding by $30-50 \%$; however, there was no significant increase of bleeding-related mortality rate except for intracranial surgery.

The patient underwent DES implantation for LAD and LCX artery stenoses, prematurely discontinued dual antiplatelet therapy and was diagnosed with diabetes mellitus as wall as low 
ejection fraction. Therefore, he was highly susceptible to stent thrombosis. The patient should have continued dual antiplatelet therapy or if needed, discontinued dual antiplatelet therapy for a shorter period of time than other patients with a low risk of stent thrombosis.

The intraoperative anesthetic management of patients with cardiac function problems should be also taken into consideration. ACCF/AHA Preoperative Guidelines [3] in 2009 reported that to use volatile anesthetic agents during noncardiac surgery can be beneficial for the patient with risk for MI to maintain general anesthesia in hemodynamically stable state, and the used of opioid-associated anesthetics can help with the cardiovascular stability. Even though all inhaled volatile anesthetic agents share cardiovascular effects including depression of myocardial contractility and afterload reduction, many randomized clinical trials among patients undergoing coronary artery bypass graft surgery verified that volatile anesthetics decrease troponin release and enhance left ventricular function, compared with propofol or midazolam, which also can be applied to patients with coronary artery disease undergoing noncardiac surgery [3]. In this case, the patient had coronary artery stents with a high cardiovascular risk including left ventricular dysfunction, so inhalation agents and opioid-associated anesthetics were used in order to minimize the fluctuation in his hemodynamic signs.

During noncardiac surgical procedures, intravenous NTG infusions are selected in many cases to prevent intraoperative MI. Coriat et al. [9] reported that NTG infusion can be beneficial in preventing intraoperative MI. Moon et al. [10] also demonstrated that intravenous NTG infusions efficiently decrease hemodynamic changes in some degree for patients in the Trendelenburg position and pneumoperitoneum during laparoscopic low anterior resections. The patient in the case report was in the Trendelenburg position with $\mathrm{CO}_{2}$ insufflation for laparoscopic surgery, but then severe ST depression occurred; therefore, NTG $0.5 \mu \mathrm{g} / \mathrm{kg} / \mathrm{min}$ was infused. After NTG infusion, ST depression steadily improved, and the operating table returned to the neutral position and the patient was eliminated from pneumoperitoneum, ST depression restored its initial status and NTG infusion was stopped because of low BP.

Sudden cardiac arrest still has a low survival rate despite introduction of CPR, with the rate remaining unchanged, since 1993 and conventional CPR has been associated with high immediate mortality [11]. Therefore, new CPR methods have been tried to increase survival rate, and among these attempts, extracorporeal life support was proposed as a device for cardiac resuscitation in the early 1960s. Extracorporeal CPR (ECPR), an alternative to conventional CPR that employs ECMO, has been reported to be effective for patients in whom the return of spontaneous circulation cannot be achieved by conventional CPR [11-13]. ECMO provides adequate temporary perfusion to organs in cardiac arrest patients before return of spontaneous heartbeat, so it has higher hospital discharge rate and short- and long-term survival rates compared with conventional CPR for complex patients $[11,14]$.

A high incidence of ACS is reported in cardiac arrest patients and it has been noted recently that the use of PCI in postresuscitation care improves their outcome [12]. Percutaneous cardiopulmonary support provides safe condition for stent coronary revascularization in patients with acute $\mathrm{MI}$ or congestive heart failure [15], so it can reduce mortality and morbidity rates, because the most effective way to treat acute MI caused by stent thrombosis is early reperfusion, so primary PCI is recommended rather than less effective thrombolytic therapy [3]. Kagawa et al. [13] reported for the early ECPR that a significantly high percentage of patients receiving intra-arrest PCI achieve return of spontaneous heartbeat. In addition, 30-day survival and favorable neurological outcome of rapid-response ECMO and PCI were higher than ones of conventional CPR [13]. Therefore, rapid-response ECMO and intra-arrest PCI can be associated with improved outcomes in patients who are unresponsive to conventional CPR, especially with ACS [13]. One reason of above results is that ECMO can provide temporary total circulatory support and increase coronary perfusion, as a result, successful defibrillation has increased $[13,15]$. Second reason is that intraarrest PCI is effective for ACS patients because higher coronary perfusion pressure is necessary for more quickly achieving return of spontaneous heartbeat [13]. As the result, emergent revascularization of occluded coronary arteries for refractory cardiac arrest patients is very important.

According to the case report, 7 minutes of CPR could not succeed in inducing the patient's heart rhythm, and based on his medical history, stent thrombosis was most suspected; as such, early ECMO insertion was considered, and it was completed 23 minutes after CPR. ECMO supported hemodynamic stability and oxygenation, so even with ventricular fibrillation and ventricular tachycardia, relatively stable vital signs allowed successful revascularization. Since conventional CPR initiated immediately after his first ventricular fibrillation played a critical bridging role until ECMO insertion, and early ECMO insertion and revascularization could treat stent thrombosis causing cardiac arrest, the patient's cardiac function could be restored at a rather fast speed. Additionally, IABP was inserted together with revascularization, because it was reported that patients undergoing ECMO together with IABP showed additional pulsatile flow, reduction of afterload, and better coronary flow [14], and IABP insertion also helped his fast recovery.

In conclusion, if patients undergoing DES prematurely discontinue dual antiplatelet therapy, occurrence rate of stent thrombosis increases, especially with risk factors, such as diabetes mellitus, low ventricular ejection fraction, and stent length. 
Therefore, noncardiac surgery should be postponed for a minimum of 12 months after DES, and if not available, dual antiplatelet therapy should continue or at least aspirin should be administered. Considering high mortality of MI induced by perioperative stent thrombosis, if stent thrombosis is suspected as a main cause of cardiac arrest, chest should be effectively compressed to circulate blood and oxygen, but if conventional CPR cannot restore a heart rhythm, early veno-arterial ECMO insertion should be considered in the earliest possible stage.

\section{References}

1. Brilakis ES, Banerjee S, Berger PB. Perioperative management of patients with coronary stents. J Am Coll Cardiol 2007; 49: 2145-50.

2. Cruden NL, Harding SA, Flapan AD, Graham C, Wild SH, Slack R, et al. Previous coronary stent implantation and cardiac events in patients undergoing noncardiac surgery. Circ Cardiovasc Interv 2010; 3: 236-42.

3. Fleisher LA, Beckman JA, Brown KA, Calkins H, Chaikof EL, Fleischmann KE, et al. 2009 ACCF/AHA focused update on perioperative beta blockade incorporated into the ACC/AHA 2007 guidelines on perioperative cardiovascular evaluation and care for noncardiac surgery: a report of the American college of cardiology foundation/American heart association task force on practice guidelines. Circulation 2009; 120: e169-276.

4. Iakovou I, Schmidt T, Bonizzoni E, Ge L, Sangiorgi G, Stankovic G, et al. Incidence, predictors, and outcome of thrombosis after successful implantation of drug-eluting stents. JAMA 2005; 293: 2126-30.

5. Rade JJ, Hogue CW Jr. Noncardiac surgery for patients with coronary artery stents: timing is everything. Anesthesiology 2008; $109: 573-5$.

6. Row D, Weiser MR. An Update on laparoscopic resection for rectal cancer. Cancer Control 2010; 17: 16-24.

7. Rabbitts JA, Nuttall GA, Brown MJ, Hanson AC, Oliver WC, Holmes DR, et al. Cardiac risk of noncardiac surgery after percutaneous coronary intervention with drug-eluting stents. Anesthesiology 2008; 109: 596-604.

8. Chassot PG, Delabays A, Spahn DR. Perioperative antiplatelet therapy: the case for continuing therapy in patients at risk of myocardial infarction. Br J Anaesth 2007; 99: 316-28.

9. Coriat P, Daloz M, Bousseau D, Fusciardi J, Echter E, Viars P. Prevention of intraoperative myocardial ischemia during noncardiac surgery with intravenous nitroglycerin. Anesthesiology 1984; 61: 193-6.

10. Moon HS, Lee SK, Choi YS, In CB, Choi EJ. The effect of nitroglycerin on hemodynamic changes during laparoscopic low anterior resection. Korean J Anesthesiol 2011; 61: 388-93.

11. Chen YS, Lin JW, Yu HY, Ko WJ, Jerng JS, Chang WT, et al. Cardiopulmonary resuscitation with assisted extracorporeal life-support versus conventional cardiopulmonary resuscitation in adults with in-hospital cardiac arrest : an observational study and propensity analysis. Lancet 2008; 372: 554-61.

12. Nagao K, Hayashi N, Kanmatsuse K, Arima K, Ohtsuki J, Kikushima K, et al. Cardiopulmonary cerebral resuscitation using emergency cardiopulmonary bypass, coronary reperfusion therapy and mild hypothermia in patients with cardiac arrest outside the hospital. J Am Coll Cardiol 2000; 36: 776-83.

13. Kagawa E, Dote K, Kato M, Sasaki S, Nakano Y, Kajikawa M, et al. Should we emergently revascularize ccluded coronaries for cardiac arrest? rapid-response extracorporeal membrane oxygenation and intra-arrest percutaneous coronary intervention. Circulation 2012; 126: 160513.

14. Bakhtiary F, Keller H, Dogan S, Dzemali O, Oezaslan F, Meininger D, et al. Venoarterial extracorporeal membrane oxygenation for treatment of cardiogenic shock: clinical experiences in 45 adult patients. J Thorac Cardiovasc Surg 2008; 135: 382-8.

15. Suárez de Lezo J, Pan M, Medina A, Pavlovic D, Romero M, Segura J, et al. Percutaneous cardiopulmonary support in critical patients needing coronary interventions with stents. Catheter Cardiovasc Interv 2002; 57: 467-75. 